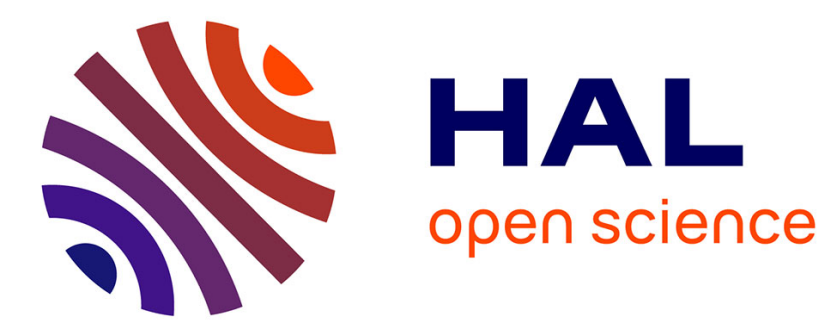

\title{
Phase field model of the nanoscale evolution during the explosive crystallization phenomenon
}

S F Lombardo, S. Boninelli, Fuccio Cristiano, I. Deretzis, M.G. Grimaldi, K. Huet, E. Napolitani, A. La Magna

\section{- To cite this version:}

S F Lombardo, S. Boninelli, Fuccio Cristiano, I. Deretzis, M.G. Grimaldi, et al.. Phase field model of the nanoscale evolution during the explosive crystallization phenomenon. Journal of Applied Physics, 2018, 123 (10), 10.1063/1.5008362 . hal-01921142

\section{HAL Id: hal-01921142 \\ https://hal.science/hal-01921142}

Submitted on 13 Nov 2018

HAL is a multi-disciplinary open access archive for the deposit and dissemination of scientific research documents, whether they are published or not. The documents may come from teaching and research institutions in France or abroad, or from public or private research centers.
L'archive ouverte pluridisciplinaire $\mathbf{H A L}$, est destinée au dépôt et à la diffusion de documents scientifiques de niveau recherche, publiés ou non, émanant des établissements d'enseignement et de recherche français ou étrangers, des laboratoires publics ou privés. 
Phase field model of the nanoscale evolution during the explosive crystallization phenomenon

S. F. Lombardo, S. Boninelli, F. Cristiano, I. Deretzis, M. G. Grimaldi, K. Huet, E. Napolitani, and A. La Magna

Citation: Journal of Applied Physics 123, 105105 (2018); doi: 10.1063/1.5008362

View online: https://doi.org/10.1063/1.5008362

View Table of Contents: http://aip.scitation.org/toc/jap/123/10

Published by the American Institute of Physics

\section{Scilight}

Sharp, quick summaries illuminating the latest physics research 


\title{
Phase field model of the nanoscale evolution during the explosive crystallization phenomenon
}

\author{
S. F. Lombardo, ${ }^{1,2, a)}$ S. Boninelli, ${ }^{3}$ F. Cristiano, ${ }^{4}$ I. Deretzis, ${ }^{2}$ M. G. Grimaldi, ${ }^{1,3}$ K. Huet, ${ }^{5}$ \\ E. Napolitani, ${ }^{3,6}$ and A. La Magna ${ }^{2, b)}$ \\ ${ }^{1}$ Dipartimento di Fisica e Astronomia, Università di Catania, Via Santa Sofia 64, 95125 Catania, Italy \\ ${ }^{2}$ CNR-IMM, Z.I. VIII Strada 5, 95121 Catania, Italy \\ ${ }^{3}$ CNR-IMM, Via S. Sofia 64, I-95123 Catania, Italy \\ ${ }^{4} L A A S, C N R S$ and University of Toulouse, 7 av. Du Col. Roche, 31400 Toulouse, France \\ ${ }^{5}$ SCREEN Semiconductor Solutions Co., Ltd., Gennevillers, France \\ ${ }^{6}$ Dipartimento di Fisica Astronomia, Università di Padova, Via Marzolo 8, 35131 Padova, Italy
}

(Received 5 October 2017; accepted 17 February 2018; published online 12 March 2018)

\begin{abstract}
Explosive crystallization is a well known phenomenon occurring due to the thermodynamic instability of strongly under-cooled liquids, which is particularly relevant in pulsed laser annealing processes of amorphous semiconductor materials due to the globally exothermic amorphous-toliquid-to-crystal transition pathway. In spite of the assessed understanding of this phenomenon, quantitative predictions of the material kinetics promoted by explosive crystallization are hardly achieved due to the lack of a consistent model able to simulate the concurrent kinetics of the amorphous-liquid and liquid-crystal interfaces. Here, we propose a multi-well phase-field model specifically suited for the simulation of explosive crystallization induced by pulsed laser irradiation in the nanosecond time scale. The numerical implementation of the model is robust despite the discontinuous jumps of the interface speed induced by the phenomenon. The predictive potential of the simulations is demonstrated by means of comparisons of the modelling predictions with experimental data in terms of in situ reflectivity measurements and ex-situ micro-structural and chemical characterization. Published by AIP Publishing. https://doi.org/10.1063/1.5008362
\end{abstract}

\section{INTRODUCTION}

Ultrafast pulsed Laser Annealing (LA) is gaining relevance in nano-manipulation processing due to the increasing necessity of strongly localized heating of nano-structures and devices. ${ }^{1}$ In many of these applications, especially those devoted to the semiconductor technology, LA is preceded by process steps (e.g., impurities' implantation or low temperature deposition) producing strongly disordered or amorphous thin film materials. Moreover, for the majority of the proposed conditions, LA induces localized melting and ultra fast recovering of the crystalline order. This process promotes the material modifications (e.g., the transition of the amorphous regions in nano/microcrystalline ones or the redistribution of the alloy relative concentration) within a few tens of nanometer thick regions close to the sample surface. Under melting conditions, the Explosive Crystallization (EC) phenomenon occurs almost ubiquitously during these processes due to the formation of a strongly under-cooled liquid layer., ${ }^{2,3}$ This phenomenon usually hinders the process control and, as a consequence, the material properties and its quality after the irradiation are difficult to predict. This difficulty is also caused by the lack of a consistent model able to simulate the concurrent kinetics of the amorphous-liquid and liquid-crystal interfaces.

In this paper, the application of a phase field (PF) formalism to the simulation of explosive crystallization is

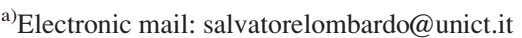

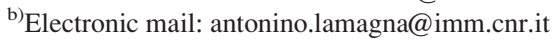

discussed. The explosive kinetics are recovered naturally by the model solutions since the unbalance of the thermodynamic parameters of the three concurring phases can be rightly implemented. The modelling results will also be validated by means of wide comparison with in-situ and ex-situ characterization of processed samples.

\section{THE MECHANISM OF EXPLOSIVE CRYSTALLIZATION}

The EC mechanism in covalent elemental semiconductors ( $\mathrm{Si}$ and $\mathrm{Ge}$ ) is known (see, e.g., Ref. 4 and references therein) as a result of a significantly larger (negative) latent heat for the liquid to crystal phase transition with respect to the one (positive) for the amorphous to liquid transition. More recently, the mechanism has undergone a thorough analysis and experimental validation also by means of micro-structural investigations. ${ }^{2,3,5}$ Without loss of generality, the mechanism is explained in Fig. 1 for a particular initial state (the amorphous layer on top of the crystal bulk) which is a schematic of the experimental samples we will analyze in the following. Due to, e.g., the laser pulse heating, the temperature in the sample grows up and the amorphous layer can melt and interface kinetics is governed by the energy and mass balance laws of the liquid-amorphous inter-phase [Fig. 1(a)]. In particular, the velocity of the melting front is of the order of few $\mathrm{m} / \mathrm{s}$.

We note that the melting of amorphous covalent semiconductors is a first order phase transition. ${ }^{6-9}$ Moreover, an out of equilibrium interface between the amorphous and liquid phases forms, although both amorphous and liquid are 
a)

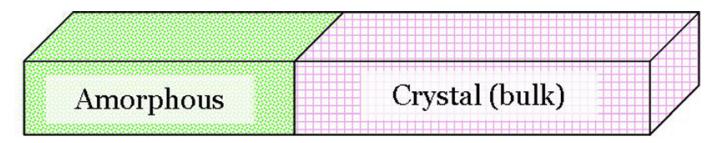

b)

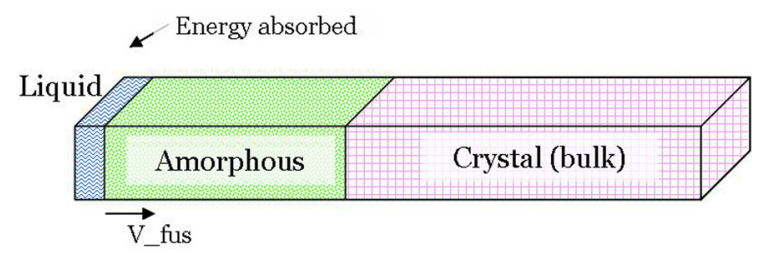

c)

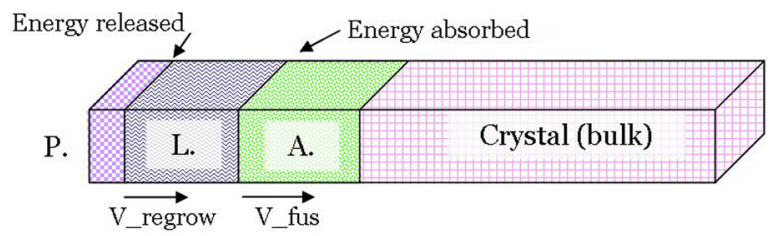

d)

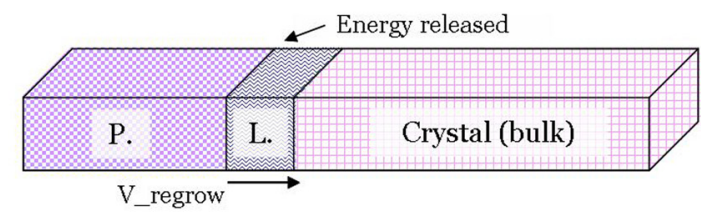

e)

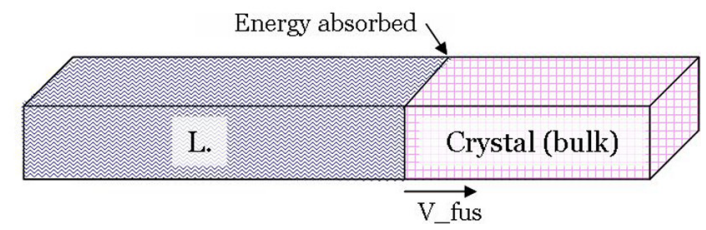

FIG. 1. Schematic of the main evolution stages of the explosive crystallization. The laser source heats the sample from the left.

meta-stable phases at atmospheric pressure in the temperature interval between the melting point of the amorphous and the melting point of the crystal (being the crystal the thermodynamic stable phase). ${ }^{10,11}$ The temperature field in the liquid phase is close to the melting point of the amorphous phase, i.e., the liquid is in an under-cooled condition with respect to the crystalline phase which in turn is more stable in this temperature range. As a consequence, the liquid portion tends to spontaneously solidify. After the nucleation time, which depends on many factors like the local temperature and the presence of impurities, the liquid solidifies and a second transition front (from liquid to crystalline) is created which is governed by crystal-liquid inter-phase characteristics. The latent heat of the solidification of the crystal is larger than the one for the melting of the amorphous. Hence, there is an excess of heat that leads to the melting of the amorphous layer that can self-sustain the phenomenon: i.e., due to this positive feedback, the process is self-propagating [Fig. 1(c)]. In this condition, the velocity of the two self-sustaining melting fronts is about one order of magnitude larger with respect to the single interface case. The fusion process ends when the energy released is insufficient to melt more amorphous material or when the polycrystalline-liquid front reaches the crystalline bulk [Fig. 1(d)]. In a 3D real system, the nucleation of the polycrystalline is a random phenomenon in space; as a consequence, the melting front spreads all over the specimen, with multiple fronts so the process could stop before reaching the amorphous/crystal interface. We would like to underline that after the first solidification event the power released by the laser is less relevant with respect to the evolution of the system, since the process is governed only by the meltingsolidification evolution. At the end of the EC phenomena, there is a nano- poly-crystalline layer upon a crystal bulk. If the external heating source is sufficiently intense, a secondary melting of the polycrystalline layer can occur in the case that the maximum of the temperature field reaches the crystal melting point [Fig. 1(e)]. This secondary liquid-crystal melt front evolves conventionally since, after the EC, the system presents only the crystal thermal properties.

\section{A PHASE FIELD MODEL FOR THE EXPLOSIVE CRYSTALLIZATION}

The modelling of the EC phenomenon should allow the computation of the contemporary kinetics of the crystal-liquid and liquid-amorphous front on the basis of the thermodynamic parameters of the substance in study. Moreover, if the heating is obtained by a laser, the self consistent evaluation of the heat source using the electromagnetic field equations are necessary for the accurate prediction of the thermal field evolution in the nano-scale. ${ }^{12}$ The potentiality of the Phase Field (PF) formalism $^{13-17}$ for the simulation of pulsed irradiation processes has been demonstrated in several papers, see Ref. 18 and references therein. PF also allows reliable extensions to compute the evolution of other quantities (e.g., impurity and defect fields ${ }^{19}$ ); therefore, it could be the ideal framework for implementing a comprehensive EC modeling. In the following, we formalize a phase field EC model where the PF variable $\phi$ assumes multiple values related to the three phases involved in the EC.

\section{A. Phase and temperature fields}

We start from the Karma-Rappel derivation ${ }^{16}$ which we briefly resume in the following reformulated for a dimensional real temperature variable. Moreover, since we deal with a strongly under-cooled liquid phase, we also consider a generic non-linear dependence of the solid-liquid interface speed on the interface temperature according to the method discussed in Ref. 20. The phase field equations for a generic temperature $T$ driven by the solid-liquid phase transition are

$$
\begin{gathered}
\tau \frac{\partial \phi}{\partial t}=W^{2} \nabla^{2} \phi-\frac{\partial F(\phi, T)}{\partial \phi}, \\
c_{p} \frac{\partial T}{\partial t}=\nabla[K(\phi, T) \nabla T]+\frac{1}{2} L \frac{\partial h(\phi)}{\partial t},
\end{gathered}
$$

where

$$
F(\phi, T)=f(\phi)+\lambda(T)\left[T-T_{M}\right] g(\phi) .
$$

The parameters of the model are $W$ the interface thickness, $\tau$ the phase field time parameter, $K$ the heat conductivity, $c_{p}$ the specific heat, $T_{M}$ the melting point, and $L$ the latent heat, while $F(\phi, \lambda u)$ and $h$ functions must be chosen in a way that 
the diffuse interface solutions coincide with the free boundary problem in the sharp interface limit

$$
\begin{aligned}
c_{p} \frac{\partial T}{\partial t} & =\nabla[K(\phi, T) \nabla T], \\
V & =K / L\left(\left.\partial_{n} u\right|^{-}-\left.\partial_{n} u\right|^{+}\right), \\
T-T_{i} & =-L / c_{p}\left(\frac{d_{0}}{R}-\beta V\right),
\end{aligned}
$$

where $d_{0}$ is the capillarity length and $\beta$ is the kinetic coefficient which relates the local interface temperature $T_{i}$ to the local interface speed. Karma and Rappel proposed a double well function with minima at $\phi=-1$ (liquid) and $\phi=+1$ (solid) using the following expressions for $f, g$, and $h$ :

$$
\begin{gathered}
f(\phi)=-\frac{1}{2} \phi^{2}\left(1-\frac{1}{2} \phi^{2}\right), \\
g(\phi)=\phi-\frac{2}{3} \phi^{3}+\frac{1}{5} \phi^{5}, \\
\frac{1}{2} \frac{\partial h(\phi)}{\partial t}=\frac{15}{16}(1+\phi)^{2}(1-\phi)^{2} \frac{\partial \phi}{\partial t},
\end{gathered}
$$

where the variational choice $h(\phi) \propto g(\phi)$ and the additional constraints

$$
\frac{h(+1)-h(-1)}{2}=1
$$

are imposed in order to guarantee that the correct amount of latent heat is produced/consumed at the moving interface. For small undercooling, $\lambda$ is a constant and the sharp interface limit fixes the $\lambda$ 's value in Eq. (3) as the one satisfying the following equation:

$$
\beta=a_{1}\left[\frac{\tau c_{p}}{\lambda L W}-a_{2} \frac{W c_{p}}{K}\right]
$$

with $a_{1}=0.8839$ and $a_{2}=0.3981$, while $d_{0}=a_{1} W c_{p} / L \lambda{ }^{16}$ Note that a nonlinear relation between $V\left(T_{i}\right)$ and the moving interface temperature $T_{i}$ can be implemented deriving an inverse function $\lambda\left[V\left(T_{i}\right)\right]$ from $\beta\left[V\left(T_{i}\right)\right]$ with the use of the (9) expression. ${ }^{20}$ In particular, we consider the expression of the Fulcher-Vogel ${ }^{21,22}$ proposed by Stiffler et al. ${ }^{23}$ for the speed of the interface $V(T)$

$$
V=A \cdot \exp \left(\frac{-E_{a}}{k_{b} T}\right) \cdot\left[1-\exp \left[\left(\frac{\rho L}{k_{b} N}\right) \times\left(\frac{1}{T_{M}}-\frac{1}{T}\right)\right]\right]
$$

which assumes that the front speed is a net result from the balance of activated transitions occurring at the interface and promoting atoms from the liquid phase to the solid one and vice-versa (the equation parameters are described and reported in Table I). We note that, according to expression 10, the speed of the crystalline-liquid interface is $\sim 16 \mathrm{~m} / \mathrm{s}$ at the melting temperature of the amorphous phase. We have numerically verified that the phase boundary speed follows the trend of Eq. (10) for iso-thermal simulations in the case of a flat [one dimensional (1D)] interface.

This formulation is capable of describing the copresence of two phases only in the system (e.g., crystal/liquid or amorphous/liquid). The idea behind our extension is the formulation of a three-phase model suitable for the EC kinet-

\begin{tabular}{|c|c|c|c|c|}
\hline Parameter & Description & Amorphous & Crystalline & Liquid \\
\hline$L(\mathrm{~J} / \mathrm{g})$ & Latent heat & 350 & 510 & 1 \\
\hline$T_{M}(\mathrm{~K})$ & Melting temperature & 965 & 1210 & I \\
\hline$C_{p}[\mathrm{~J} /(\mathrm{g} \mathrm{K})]$ & Thermal capacitance & $1.72 \times 10^{-4} \cdot T+0.29$ & $1.17 \times 10^{-4} \cdot T+0.293$ & 0.46 \\
\hline$\rho\left(\mathrm{g} / \mathrm{cm}^{3}\right)$ & Density & 5.32 & 5.32 & $5.6-0.625 \times 10^{-3} \cdot\left(T-T_{M c}\right)$ \\
\hline$K[\mathrm{~W} /(\mathrm{cm} \mathrm{K})]$ & Thermal conductivity & 0.158 & $0.602 \cdot\left(\frac{T}{300}\right)^{-1.25}$ & 0.297 \\
\hline$A(\mathrm{~m} / \mathrm{s})$ & Speed prefactor & $5 \times 10^{4}$ & $0.3 \times 10^{4}$ & l \\
\hline$E_{a}(\mathrm{eV})$ & Speed activation energy & 0.52 & 0.5 & / \\
\hline$N\left(1 / \mathrm{cm}^{3}\right)$ & Atomic density & $4.15 \times 10^{22}$ & $4.56 \times 10^{22}$ & / \\
\hline$n$ & Index of refraction & 2.7 & 3.993 & 1.18 \\
\hline$k$ & Extinction coefficient & 2.77 & 3.398 & 3.398 \\
\hline $\mathrm{n}_{\text {probe }}$ & Index of refraction & 4.667 & 5.44 & 3.26 \\
\hline $\mathrm{k}_{\text {probe }}$ & Extinction coefficient & 1.362 & 0.691 & 5.92 \\
\hline $\mathrm{D}\left(\mathrm{cm}^{2} / \mathrm{s}\right)$ & Diffusion coefficient & $8.7 \times 10^{7} \exp \left(\frac{-3.46}{k_{B} \cdot T}\right)$ & $8.7 \times 10^{7} \exp \left(\frac{-3.46}{k_{B} \cdot T}\right)$ & $D_{P}^{H D}=0.21 \cdot \exp \left(\frac{-0.6}{k_{B} \cdot T}\right)$ \\
\hline & & & & $D_{P}^{L D}=4.2 \times 10^{-3} \cdot \exp \left(\frac{-0.6}{k_{B} \cdot T}\right)$ \\
\hline \multirow[t]{2}{*}{$\mathrm{k}_{\text {out }}$} & Out diffusion coefficient & $2.8 \times 10^{2} \exp \left(\frac{-0.8}{k_{B} \cdot T}\right)$ & $2.8 \times 10^{2} \exp \left(\frac{-0.8}{k_{B} \cdot T}\right)$ & $\mathrm{HD}=0.47$ \\
\hline & & & & $\mathrm{LD}=0.04$ \\
\hline
\end{tabular}
ics. The first step is a re-scaling of the phase function as

$$
\phi \rightarrow \frac{1+\phi}{2}
$$

in order to use the positive sector of the phase values $\phi \geq 0$ for the crystal/liquid phase balance, similarly to the Wheeler's phase field formulation. ${ }^{24,25}$ Moreover, we extend the physical meaning of the phase parameter to the negative sector, where

TABLE I. Data parameters of germanium. 
negative values of the phase rule the amorphous/liquid interphase, while the crystal/liquid one is governed by the positive values. As a consequence, in order to suitably modify the $\mathrm{PF}$ equations, in addition to scaling Eq. (11), we also change the expression for the potential energy density [Eq. (5)] as follows:

$$
f(\phi) \rightarrow \tilde{f}(\phi)=-\phi^{2}(1-|\phi|)^{2} .
$$

Consistently, the derivatives of the drift terms of phase and temperature equations are replaced by

$$
\frac{\partial \tilde{g}}{\partial \phi}=8 \phi^{2}(1-|\phi|)^{2}
$$

and

$$
\frac{1}{2} \frac{\partial \tilde{h}_{2}(\phi)}{\partial t}=30 \phi^{2}(1-|\phi|)^{2} \frac{\partial \phi}{\partial t}
$$

This formulation exploits three local minima of the potential energy density at $-1,0,1$ (see the orange curve in Fig. 2 compared to the potential energy of the original two phase formalism shown as a green line). One of these minima [the global minimum of $F(\phi, T)]$ is related to the stable phase, while the other two are related to the metastable ones, where the stability of one phase with respect to the others is ruled by the local temperature through the modified Eq. (3) for the total free energy density.

The last part of the modelling extension is related to the modifications of the expressions for the thermodynamic parameters of the model which now implements a two sector (negative and positive $\phi$ ) dependence to consider properly the values for the amorphous/liquid and crystal/liquid case. Consequently, the complete PF for the EC reads

$$
\begin{aligned}
\tau \frac{\partial \phi}{\partial t}= & W^{2} \nabla^{2} \phi-\frac{\partial \tilde{f}}{\partial \phi}-\lambda(T, \phi)\left[T-T_{M}\right] \frac{\partial \tilde{g}}{\partial \phi} \\
= & W^{2} \nabla^{2} \phi-2 \phi(1-|\phi|)(1-2|\phi|) \\
& -8 \lambda(T, \phi)\left[T-T_{M}\right] \phi^{2}(1-|\phi|)^{2},
\end{aligned}
$$

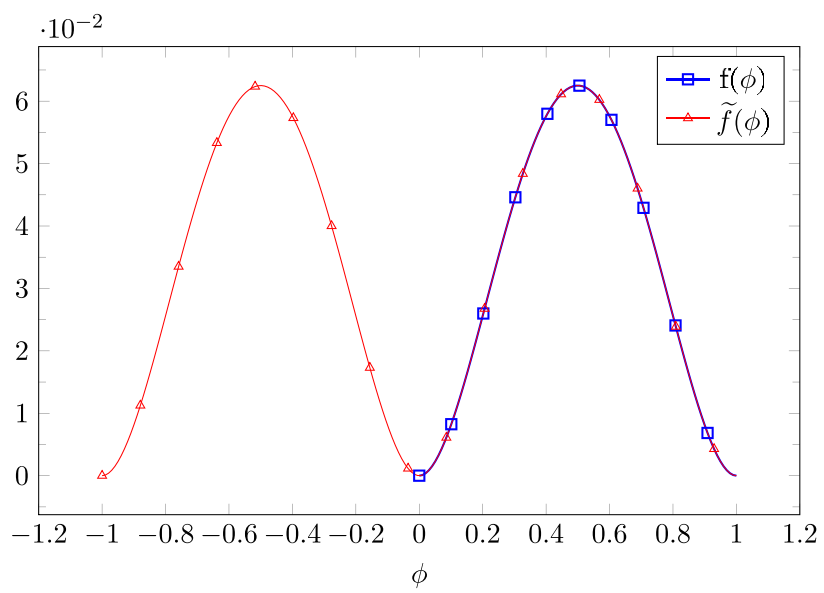

FIG. 2. Potential energy function $f(\phi)$ of the Wheeler formulation with two minima (blue curve and squares) and the one used in our extension with three minima (red curve and triangles).

$$
c_{p} \frac{\partial T}{\partial t}=\nabla[K(\phi, T) \nabla T]+30 L(\phi) \phi^{2}(1-|\phi|)^{2} \frac{\partial \phi}{\partial t}+S,
$$

where $S$ is the heat source which has to be self-consistently evaluated by coupling time harmonic Maxwell equations with the model (see Ref. 18 for details). Self-consistency is mandatory since, even in quasi one dimensional geometry, the laser energy during the EC is absorbed by multi-layers (e.g., crystal/liquid/amorphous layers with different optical constants) with thicknesses which could rapidly change with the time. The expressions for the coupling parameter, latent heat, melting temperature, specific heat, and thermal conductivity are, respectively,

$$
\begin{gathered}
\lambda(\phi)=\theta(\phi) \lambda_{c}(T)+[1-\theta(\phi)] \lambda_{a}(T), \\
L(\phi)=\theta(\phi) L_{c}-[1-\theta(\phi)] L_{a}, \\
T_{M}(\phi)=\theta(\phi) T_{M c}+[1-\theta(\phi)] T_{M a}, \\
C_{p}(\phi, T)=\theta(\phi) C_{p c}(T)+[1-\theta(\phi)] C_{p a}(T) \\
+C_{p l}(T)[1-|\phi|], \\
K(\phi, T)=\theta(\phi) K_{c}(T)+[1-\theta(\phi)] K_{a}(T) \\
+K_{l}(T)[1-|\phi|],
\end{gathered}
$$

where $\theta(\phi)$ is the Heaviside step function, while the subscripts $c, a$, and $l$ indicate crystal, amorphous, and liquid phases, respectively. Note that the minus sign in the latent heat expression Eq. (18) is related to the opposite time derivative of the phase function in the amorphous to liquid and crystal to liquid phase transitions.

\section{B. Dopant/impurity field}

Due to its relevance for technological applications, the possibility to predict the redistribution of impurity (or dopant) density during LA is an important feature of the LA modelling tool. Moreover, the comparison between experimental and simulated dopant profiles is a reliable method for the model validation. In particular, implanted impurities redistribute due to the high diffusivity in the liquid state and are trapped in the solid phase by the rapid re-growth. ${ }^{26}$

Extensions of phase field models ${ }^{18,25,27}$ allow the modeling of the nonequilibrium impurity trapping phenomenon which promotes a reduced segregation. For finite interface velocity $V$, non-equilibrium trapping is characterized by the solute segregation coefficient $k_{\text {segr }}(V)$, which is dependent on $V$ and tends to 1 (i.e., the no segregation case) for very high $V$, i.e., the conditions achieved during the explosive crystallization phenomenon. Several works (see, e.g., Ref. 18 and references therein) have demonstrated a non-fickian anomalous diffusion of impurities like $\mathrm{B}$ and $\mathrm{P}$ in liquid elemental semiconductors ( $\mathrm{Si}$ and $\mathrm{Ge}$ ). These anomalous effects characterize impurity diffusion even when $k \sim 1$ is expected. As a consequence, in order to accurately simulate impurity redistribution during EC, we have coupled the multi-phase field model with the two component diffusion model of Ref. 28, where non-fickian diffusion is recovered as an effect of the presence of two states of the dopant atoms with very different mobilities related to the mixed metallic-covalent bonding 
character in $l$-Si and $l$-Ge. ${ }^{29-31}$ The diffusion model in liquid is formulated as follows: ${ }^{28}$

$$
\begin{gathered}
\frac{\partial c}{\partial t}=\frac{\partial c^{H D}}{\partial t}+\frac{\partial c^{L D}}{\partial t}, \\
\frac{\partial c^{H D}}{\partial t}=\nabla\left[D^{H D} \nabla c^{H D}\right]+k^{\tau}\left(c^{L D}-\bar{R} \cdot c^{H D}\right), \\
\frac{\partial c^{L D}}{\partial t}=\nabla\left[D^{L D} \nabla c^{L D}\right]-k^{\tau}\left(c^{L D}-\bar{R} \cdot c^{H D}\right),
\end{gathered}
$$

where $D^{H D}$ and $D^{L D}$ are the impurity diffusivity in the higher and lower diffusivity state in the liquid phase. $c^{H D}$ and $c^{L D}$ are the corresponding concentrations. $\bar{R}(T)$ is the average (equilibrium) ratio between low and high diffusivity states at constant $T$. In the solid phase, metallic bonds are not present and $D_{s o l}=D_{\text {sol }}^{H D}=D_{\text {sol }}^{L D} ; k^{\tau}$ is a rate coefficient ruling the transition between the two states which should also be related to the rapidity of the bonding order fluctuations in $l$-Si and $l-\mathrm{Ge}$. In our simulation, $k^{\tau}=1.3 \times 10^{7} 1 / \mathrm{s}$. We assume that the diffusivity in the solid phases $D_{\text {sol }}$ is the same for the crystal and amorphous cases. $^{32}$

Out-diffusion from the surface to the air could also occur with some relevance during the interval dominated by the liquid phase. In our model, out-diffusion is governed by the following equation for the boundary condition at the surface position:

$$
\left.\frac{\partial C}{\partial x}\right|_{0}=k \cdot C .
$$

In Table I, we report the parameters used in the simulation. We note that some parameters depend on $T$ and they are all phase dependent.

\section{EXPERIMENTAL}

In order to properly present the model results by means of a real application case, we apply the formalism to study LA processes in implanted amorphous $\mathrm{Ge}(\alpha-\mathrm{Ge})$ on crystalline $\mathrm{Ge}(c-\mathrm{Ge})$. The experimental procedure begins with a standard cleaning on high resistivity $(0.059-0.088 \Omega \mathrm{cm})$ p-type $\mathrm{Ge}$ wafers (100), and then continues with a $\mathrm{P}$ implantation process at the energy of $15 \mathrm{keV}$ up to the dose of $1 \times 10^{15} \mathrm{~cm}^{-2}$. The implantation creates an amorphous layer, which is about 30-35 nm thick, as shown by the Transmission Electron Microscopy (TEM) analysis in the section view shown in Fig. 3. We note that an amorphous film with a not-perfectly flat amorphous/crystal interface is visible through the different contrast with respect to the underlying crystal substrate.

After the implantation, a single pulse Laser Thermal Annealing is performed (wave length $\lambda=308 \mathrm{~nm}$ ) using a SCREEN-LASSE LT system. The fluences considered for the process are $0.55,0.8$, and $1.2 \mathrm{~J} / \mathrm{cm}^{2}$. We note that, as we will discuss in the following, the $0.55 \mathrm{~J} / \mathrm{cm}^{2}$ case is the threshold energy for the melting of the $\alpha$-Ge. The coverage area of the laser spot is $\sim 1 \times 1 \mathrm{~cm}^{2}$ over $\sim 160 \mathrm{~ns}$ of exposure time of the samples to the laser pulse.

In situ Time-Resolved Reflectivity (TRR) analysis, using a laser probe at a wavelength of $635 \mathrm{~nm}$, has been applied

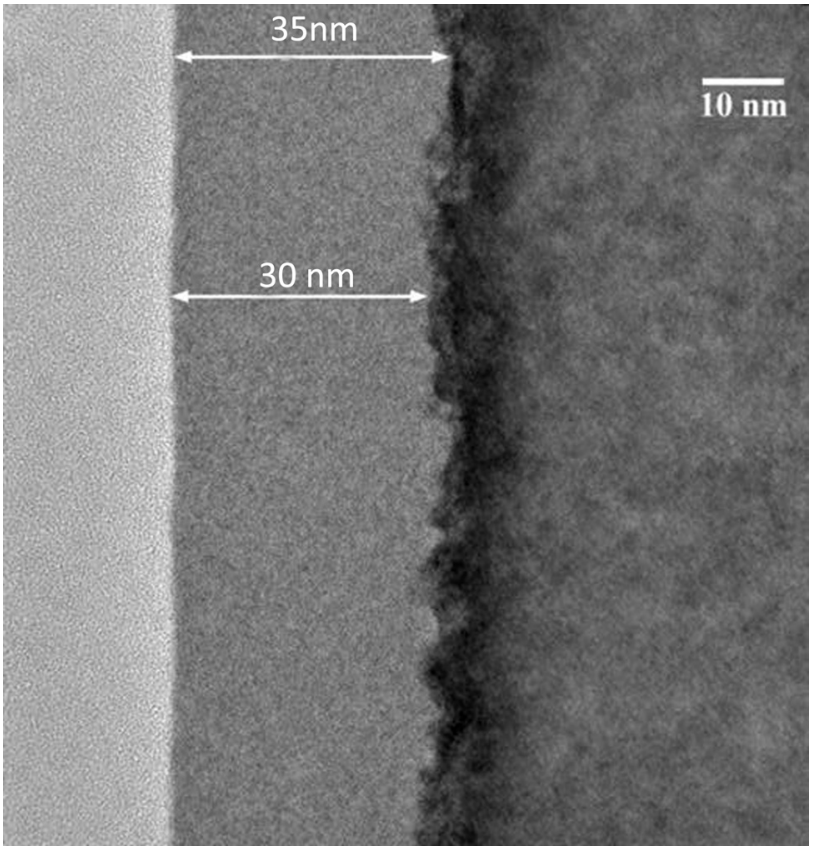

FIG. 3. TEM image of the sample before the laser process. The amorphous layer ranges between 30 and $35 \mathrm{~nm}$.

to analyse the surface optical response during the laser irradiation. After all the LA processes, the samples have been investigated by Scanning Electron Microscopy (SEM) to investigate surface quality. SEM analysis (not shown) does not reveal any particular surface features like wrinkles or ripples. TEM has been applied to control the quality of the crystal before and after the LA. Secondary Ion Mass Spectrometry (SIMS) measurements of $\mathrm{P}$ chemical profiles before and after LA have been performed using a Cameca IMS-4f instrument with a $\mathrm{Cs}+5.5 \mathrm{keV}$ primary beam.

\section{THREE PHASE SIMULATIONS}

In this section, we discuss in detail one dimensional (1D) simulations of the experimental processes presented in Sec. IV. The simulation represents a $1 \mathrm{D}$ line section of the sample with the surface on the left. The initial phase variable (see Fig. 4) matches the TEM section of the as implanted sample in Fig. 3: The top layer of $35 \mathrm{~nm}$ is in the amorphous phase, with value $\phi=-1$ of the phase variable in our method, upon a crystalline bulk up to $50 \mu \mathrm{m}$ (the substrate thickness) with a value of 1 of $\phi$. We note that we have initialized the thickness of the amorphous film at the maximum value visible from the contrast in Fig. 3, i.e., we assume that the crystal phase is positioned below the strongly damaged zone evidenced by TEM. The inset in Fig. 4 represents the laser power pulse used in all the simulations. The initial temperature of the sample is set at the value of $300 \mathrm{~K}$ in the whole simulation box. The as-implanted P SIMS profile is also shown in Fig. 4 and it is used for the initialization of the impurity density field in the LA simulations.

In Fig. 5, we plot for different times, snapshots of phase, temperature, and dopant fields obtained in the simulation of LA at a fluence of $1.2 \mathrm{~J} / \mathrm{cm}^{2}$ (similar conventions are used in these figures for indicating field types and values). The 


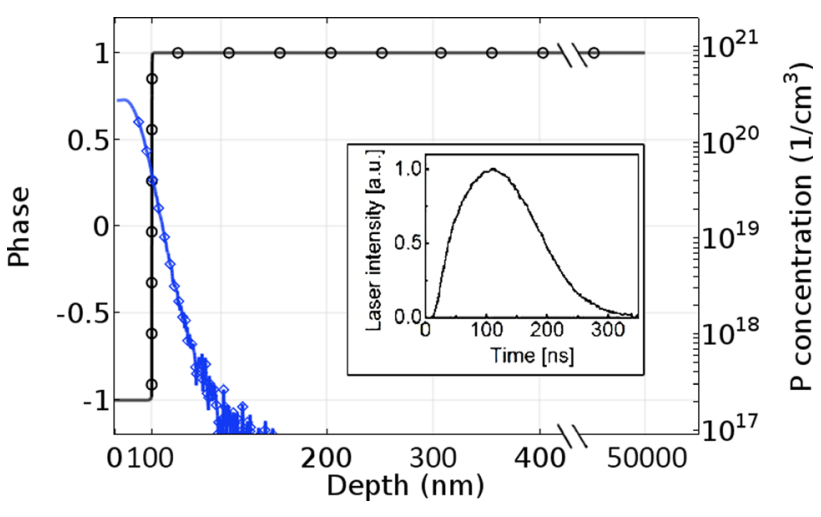

FIG. 4. Simulation $t=0 \mathrm{~ns}$. The black curve indicates the initial phase field with the value $\phi=-1$ that represents the amorphous layer, while the value $\phi=1$ is the crystalline phase. The amorphous layer is $35 \mathrm{~nm}$. The dashed blue curve is the as-implanted phosphorous concentration. Inset: the normalized laser power pulse.

nucleation radius $r_{n}$ of the (poly)crystal phase nucleating in the undercooled liquid $\mathrm{Ge}$ is assumed as $r_{n}=5 \mathrm{~nm}$ for the three cases. It is noteworthy that the amorphous/crystal interface remains frozen for temperatures below the melting point of the $\alpha$-Ge (i.e., during the initial heating), consistent with the expected activated type $\alpha-\mathrm{Ge}$ to $c$-Ge solid phase transition occurring at much larger time scales. This physical behavior is a general feature of the model implemented. Indeed, the only moving boundaries in our phase field model are the $-1 / 0$ and $0 / 1$ interfaces and the fact that there is no real liquid region (i.e., a plateau larger than $\mathrm{W}$ of the phase function at the $\phi=0$ value) at the amorphous-crystal interface does not allow any movement of the boundary. Figure 5 (a) shows the simulation results after $\mathrm{t}=155 \mathrm{~ns}$ before the occurrence of the EC nucleation event. We can observe that the simulated evolution at this time reflects the situation shown in Fig. 1 (i.e., the initial melting of the amorphous film): There is the liquid phase on the left side $(\phi=0)$, the amorphous one in the middle $(\phi=-1)$, and the crystalline in the bulk $(\phi=1)$. The speed of the amorphous-liquid interface is in the range $V \sim 2-3 \mathrm{~m} / \mathrm{s}$ in this stage. The temperature exhibits the typical diffuse decay in the bulk and a plateau (at temperatures slightly larger than the melting point of $\alpha-\mathrm{Ge}$ ) close to the surface due to both the latent heat absorption and the presence of the liquid phase with its high thermal conductivity. In Fig. 5(b), we show a snapshot corresponding to situation (c) of Fig. 1. From left to right, the phase (black curve) starts with value 1, i.e., the crystal phase, and then drops to the 0 value which is the cushion of the liquid phase (this layer is about $15 \mathrm{~nm}$ thick) and after the liquid layer, the value -1 is reached for the remaining part of the amorphous phase. The last part of the phase function is the original crystalline bulk $(\phi=1)$. We note that the real morphology of the crystal arising from the primary EC event is of poly-crystalline type. However, the current model setting does not distinguish the crystal from the poly-crystal: they share the same thermodynamic parameters and phase $(\phi=1)$ values. The red curve represents the temperature of the specimen in this stage and it shows (from left to right): a significantly higher value with respect to the melting point of $\alpha$-Ge due to the solidification on the surface (i.e., the heat is released); a descending value in the liquid layer; a lower value close to the liquid/amorphous interface (where heat is absorbed) and beyond the amorphous/crystal interface due to the typical diffusion decay. The primary front activated by the EC consumes the amorphous [see panel (d) of Fig. 1] film which is replaced by a nano-crystalline one at the end of the three phase EC phenomenon after a very short time interval [EC(time) $\sim 1 \mathrm{~ns}$ ], since the speed of the composite interface is very high $V \sim 15-16 \mathrm{~m} / \mathrm{s}$ during the EC.

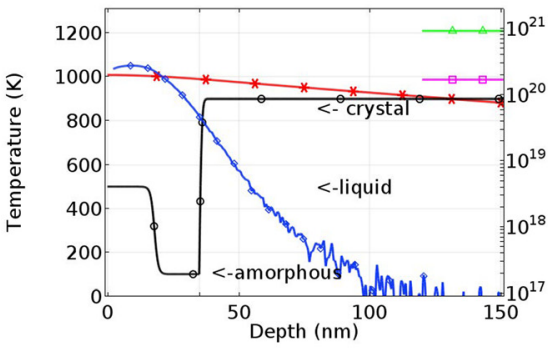

(a)

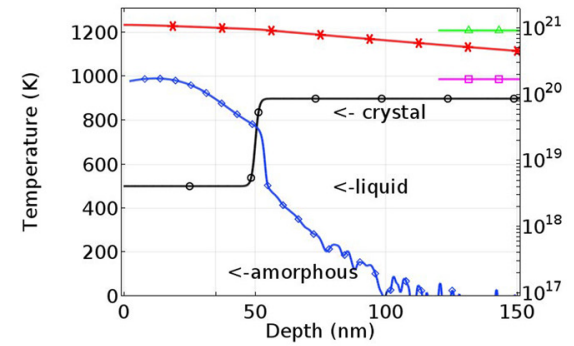

(c)

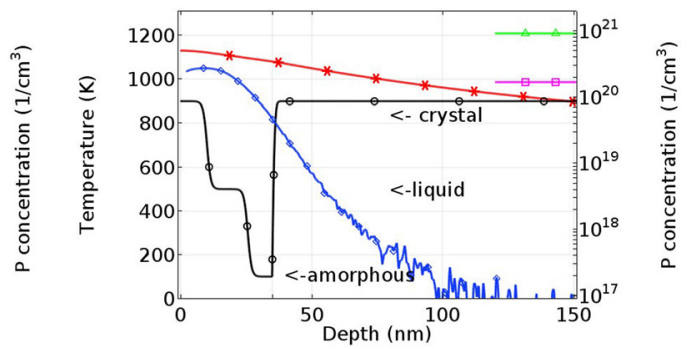

(b)

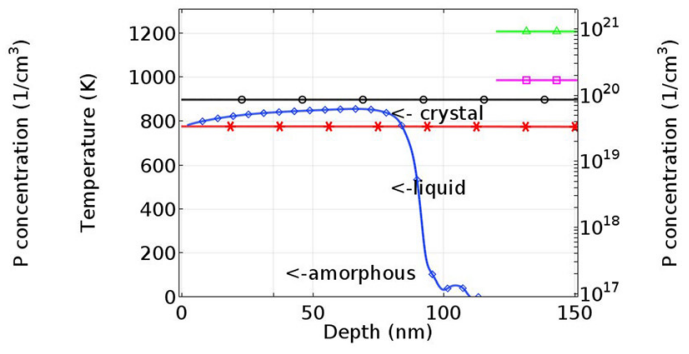

(d)

FIG. 5. Snapshots of the simulation at different times for the $1.2 \mathrm{~J} / \mathrm{cm}^{2}$ fluence case. The scale on the left is for the temperature values (red solid line and asterisk), the one on the right for the dopant density (blue solid line and rhombus), and a text description is reported for the phase (black solid line), representing the 3 main values of the $\phi$ function: $-1,0$, and +1 corresponding to the amorphous, liquid, and crystalline phases, respectively. The melting temperatures of the amorphous (magenta segment and squares) and crystal phase (green segment and triangles) are also indicated. (a) Snapshot of the simulation at $\mathrm{t}=155 \mathrm{~ns}$ (before the EC event). (b) Snapshot of the simulation at $\mathrm{t}=156 \mathrm{~ns}$ (EC event). (c) Snapshot of the simulation at $\mathrm{t}=210 \mathrm{~ns}$ (secondary melting). (d) Snapshot of the simulation at $\mathrm{t}=500 \mathrm{~ns}$ (final state). 


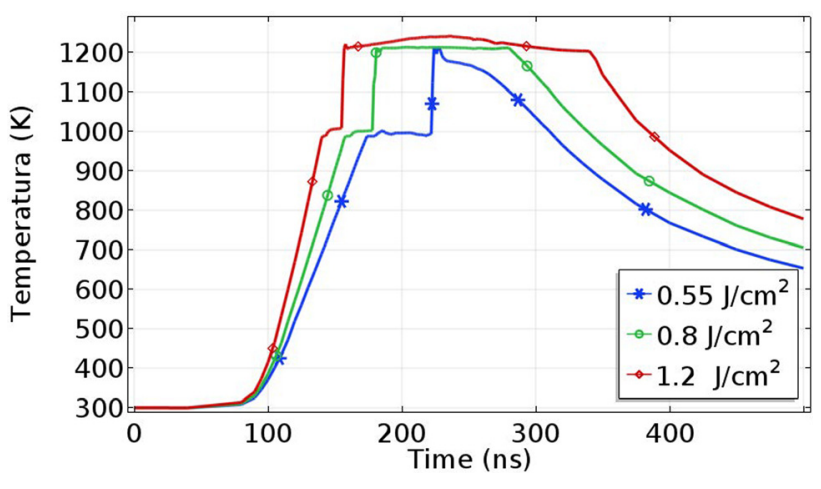

FIG. 6. Temperature at the surface for 0.55 (blue line and stars), 0.8 (green line and circles), and 1.2 (red line and rhombus) $\mathrm{J} / \mathrm{cm}^{2}$ case. Heating, fusion, and EC are present in both curves, secondary melting in the 0.8 and $1.2 \mathrm{~J} / \mathrm{cm}^{2}$ cases only.

The condition of the schematic at the panel (e) of Fig. 1 is reproduced by the simulated evolution at $\mathrm{t}=210 \mathrm{~nm}$ [see Fig. 5(c)]. A secondary (two-phase) front starts to evolve as soon as the (poly)crystal surface reaches the $c$-Ge melting point, and for these irradiation conditions, it reaches a melt depth beyond the original amorphous/crystal interface. The interface speed is about $V \sim 2-3 \mathrm{~m} / \mathrm{s}$ during the secondary melting stage and about $V \sim 1-2 \mathrm{~m} / \mathrm{s}$ during the definitive crystalline regrowth.

Figure $5(\mathrm{~d})$ shows the simulation at $\mathrm{t}=500 \mathrm{~ns}$ when the evolution of the phase and dopant fields is practically quenched. Here, the sample is fully crystalline $(\phi=1)$. The temperature, the red curve in the figure, shows a rather uniform value in the region of interest and it continues to decrease with time recovering the $300 \mathrm{~K}$ value after few tens of $\mu$ s. We underline that key-features shown by the larger fluence case are also present in the other cases, which are not presented here for brevity. The secondary front evolves only if the laser energy density is high enough to heat the surface at the $c$-Ge melting point. Otherwise, only the EC is activated as it can be inferred from Fig. 6, which shows the temperature at the surface as a function of the simulated time for the $0.55,0.8$, and $1.2 \mathrm{~J} / \mathrm{cm}^{2}$ cases in blue, green, and red, respectively. The curves share the same left part, where there is the heating (with a slope depending on the laser fluence) and the plateau due to amorphous melting. In all cases, after the nucleation time, the EC occurs with the typical signature of its spike of heating due to its extremely fast kinetics. For the lower fluence case, after the EC there is only a redistribution of the heating and then the cooling. In the 0.8 and $1.2 \mathrm{~J} / \mathrm{cm}^{2}$ cases, after the $\mathrm{EC}$ the temperature continues to rise due to the higher fluence, there is the creation of a secondary melting. In these cases, another melting condition happens at the $c$-Ge melting point because, after the EC, the surface layer has crystalline characteristics. For the $1.2 \mathrm{~J} / \mathrm{cm}^{2}$ case, the temperature increases at the surface in secondary melting and the liquid state is more pronounced, whilst for the $0.8 \mathrm{~J} / \mathrm{cm}^{2}$ case, $T$ stays close to the $T_{M}(c-\mathrm{Ge})=1210 \mathrm{~K}$ value. After the melting, there is the cooling of the sample, like in the previous case. The maximum melt depth of the secondary melt front is 15 and $84 \mathrm{~nm}$ for the 0.8 and $1.2 \mathrm{~J} / \mathrm{cm}^{2}$ cases, respectively, i.e., in the first case this value is lower than the original $\alpha-\mathrm{Ge} / c-\mathrm{Ge}$ interface. We underline that the duration of the plateau during the melting of the amorphous is different in the 3 cases; in particular, it is smaller for higher fluence. This difference is due to the different nucleation time that depends essentially on the temperature. Higher (lower) temperature, resulting from higher (lower) fluence, leads to a smaller (larger) nucleation time.

\section{MODEL VALIDATION}

In order to demonstrate the reliability of the multi-phase field EC model, we will discuss in this section the comparison between the simulation predictions and the in-situ and ex-situ experimental analyses of processed samples.

\section{A. In-situ transient reflectivity and micro-structural analysis}

In Fig. 7, we compare our simulation with in situreflectivity and TEM for the three fluences (upper panel $0.55 \mathrm{~J} / \mathrm{cm}^{2}$, middle panel $0.8 \mathrm{~J} / \mathrm{cm}^{2}$, and lower panel $1.2 \mathrm{~J} /$ $\mathrm{cm}^{2}$ ). The reflectivity is measured with a laser probe with a wavelength of $635 \mathrm{~nm}$. As a consequence, we have calculated the (multi-layer) system reflectivity at this wavelength using the time dependent phase function in order to distinguish the amorphous, liquid, and crystal layers' thicknesses. In the graphs, we plot the measured reflectivity (blue line), the calculated reflectivity (red line), whilst on the right axes the relative values of reflectivity of amorphous, crystal, and liquid $\mathrm{Ge}$ at $635 \mathrm{~nm}$ are also reported. We note that the experimental instrumentation can measure only the relative values of the reflectivity. As a consequence, the left scale is in arbitrary units. Moreover, the probe spot is not fully aligned and focused to the region heated by the main laser, so the measured value is an average of the reflectivity on a probe surface area ( $\mathrm{mm}$ scale diameter) and not a point wise value. As a consequence, we also expect some case to case variance of the reflectivity modulation if all the probed surface region does not undergo instantly the phase transitions. In the case of Figs. 7(a) and 7(b) $\left(0.55 \mathrm{~J} / \mathrm{cm}^{2}\right)$, the simulation predicts a pure EC event and, indeed, reflectivity and TEM measurements clearly indicate that this is the case. Both simulation and measurements show a single asymmetric peak of the reflectivity due to the $\alpha$-Ge melting (smooth increase) and ultrafast EC (sharp decrease), while the difference between the left and right plateau is related to the two different reflectivity values of the initial $(\alpha-\mathrm{Ge})$ and final $(c-\mathrm{Ge})$ phases. In this and in the other cases, the shape of simulated and measured reflectivity peaks is different. However, these differences can be understood in terms of the lateral propagation of the phase transition phenomena in the surface probed by the $635 \mathrm{~nm}$ laser. Indeed, while the 1D simulation assumes a single global transition event and that the propagation of the conventional melting and EC phenomena occurs only in depth, in real samples an additional concentric propagation of the front seen by the probe at the surface should occur (also due to point to point variation of the surface temperature). This lateral propagation could explain the delays observed in the experimental peaks in all the cases considered. Figure 7(b) shows the TEM image of the specimen at the end of the $0.55 \mathrm{~J} / \mathrm{cm}^{2}$ process: A polycrystalline layer with very small 


\section{In situ reflectivity}
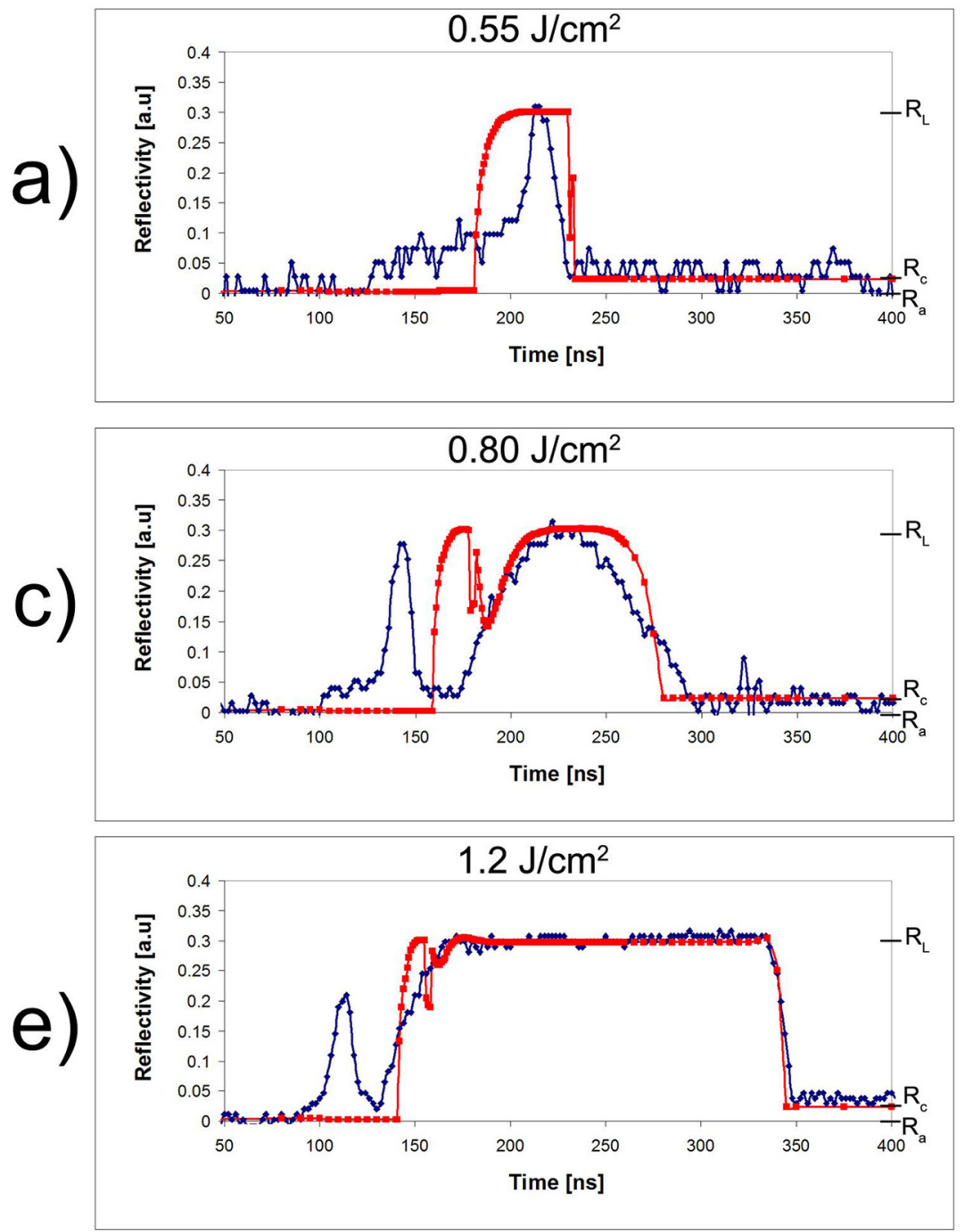

- Experiment - Simulation

\section{TEM images}
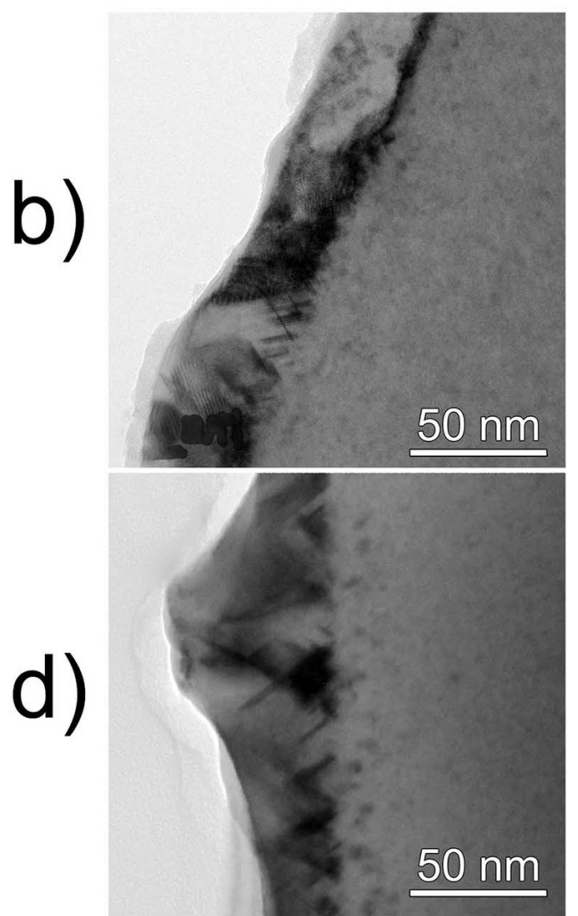

f)

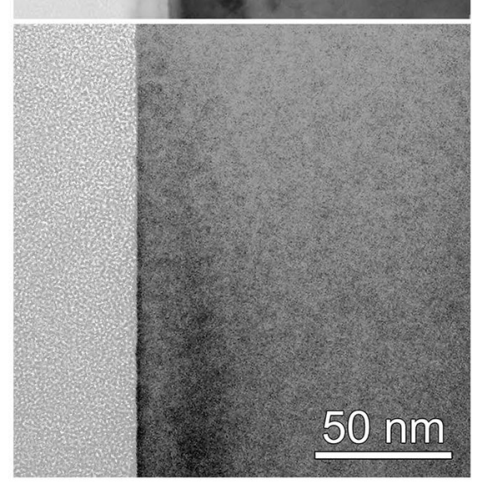

FIG. 7. Comparison between in situ reflectivity and simulation on the left and the corresponding TEM images on the right. From the top to the bottom, 0.55 , 0.8 , and $1.2 \mathrm{~J} / \mathrm{cm}^{2}$ cases are shown, respectively.

grains and a rather smooth surface replaces the original amorphous one as we expect for a pure primary EC event.

In Figs. 7(c) and 7(d), the experimental analysis refers to the case where a secondary melting front starts but it does not reach the original a/c interface. Again, the comparisons are fully consistent. The experimental and simulated reflectivities show a double feature related to the primary and secondary melting and the agreement is good in consideration of the delayed increase and decrease of the experimental curve already discussed. The TEM image at Fig. 7(d) shows a polycrystalline layer with large and inhomogeneous grains and a rough surface as we could expect from a secondary slower melting of a nano-crystalline film which does not reach the ordered crystal surface of the substrate.

Figures 7(e)-7(f) report the case with the higher value of fluence and we observe a spike related to the EC and a subsequent longer $(\sim 180 \mathrm{~ns})$ secondary melting phenomenon. In this case, the simulation predicts that the melt depth is behind the initial amorphous/crystalline interface. As a consequence, we could expect an epitaxial regrowth after the secondary melting. Indeed, the agreement is good also in this case since the TEM image shows a total defect free crystal regrowth and an extremely flat surface. In all cases, at the end of the LA, the sample shows always a crystalline (epitaxial or polycrystalline) structure everywhere and that is again in agreement with our simulations.

\section{B. Chemical analysis}

The final diffused profile of impurities is a standard integrated quantity used to study microstructural evolution during conventional laser annealing processes. Here, we discuss by comparison with EC model predictions the phosphorous redistribution in $\mathrm{Ge}$ due to the process and the experimental 


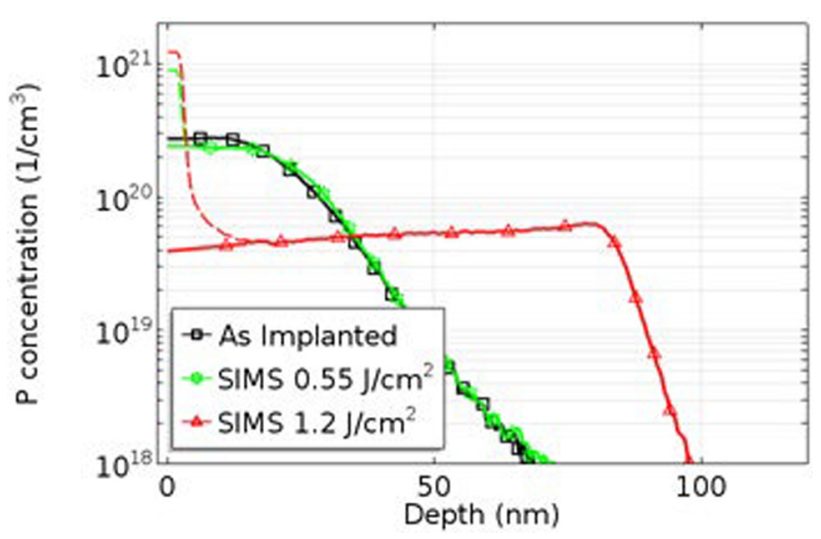

FIG. 8. Secondary ion mass spectroscopy measurement on Germanium doped with Phosphorus. The as implanted profile is plotted as a black line and squares, the profile processed with a pulse at $0.55 \mathrm{~J} / \mathrm{cm}^{2}$ as a green line and stars, and the one processed with a pulse at $1.2 \mathrm{~J} / \mathrm{cm}^{2}$ as a red line and triangles.

analysis presented in Sec. IV. We use the $\mathrm{P} / \mathrm{Ge}$ calibration of the two species diffusion model of Ref. 33 reported in Table I.

In Fig. 8, the $\mathrm{P}$ profile of the implanted sample (black and squares) and that obtained for the lower (green with stars) and higher fluence (red with triangles) cases (0.55 and $1.22 \mathrm{~J} / \mathrm{cm}^{2}$ ) are shown. The diffused profile obtained for the $1.22 \mathrm{~J} / \mathrm{cm}^{2}$ (case) shows an against gradient behavior close to the maximum melt depth position which justifies the use of the two species model specifically suited to predict anomalous redistribution of impurities during LA. Figure 9 shows the comparison between experimental and simulated profiles for the $0.55 \mathrm{~J} / \mathrm{cm}^{2}$ case. The plotted curves are the as implanted profile (black and squares), the experimental diffuse profile at end of the process (green line and stars), and the simulated diffuse profile (blue line and rhombus). In this case, as we have already discussed, only a primary EC occurs and the dopant profile beyond the initial amorphous/ crystalline interface is unchanged, since the movement of liquid layer driving the EC stops at this boundary. A limited but detectable diffusion can be observed caused by the ultrafast sweeping of the liquid layer during the EC and this impurity diffusion is also predicted by the simulation. The agreement

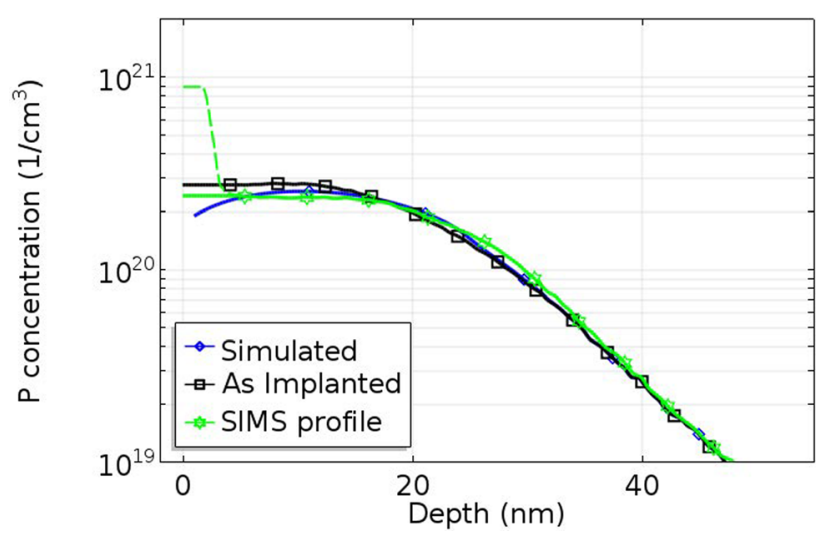

FIG. 9. Comparison between SIMS and simulation for the $0.55 \mathrm{~J} / \mathrm{cm}^{2}$ case. The black line with squares is the as implanted, green with stars is the SIMS, and solid blue and rhombus is the simulated concentration. between the simulated and SIMS profiles is good except in the surface region (about $\sim 5 \mathrm{~nm}$ from the air/sample boundary) where the SIMS measurement loses reliability. Figure 10 shows a similar analysis for the higher fluence case with the as implanted profile (in black and squares), the experimental diffuse profile (in red and triangles) at end of the process, and the simulated diffused profile (in blue with rhombus). In this case, the secondary melting occurs at a melt depth well beyond the original $\alpha-c$ interface. The combination of our model for EC and the two-state model for the impurity diffusion permits to obtain a good agreement between the simulated and experimental curves. Also in the intermediate case, the model is able to predict the distribution of the dopant (not shown). Both SIMS resolution near surface and surface roughness may compromise the comparison with simulation results.

\section{DISCUSSION}

LA is a particular process that is used in the semiconductor industry in order to achieve new features for the more and more miniaturized and complex device design. The capability to interact only with a localized area with a well controllable depth, the dopant activation capabilities, and the ultra-fast (sub- $\mu$ s) process are the keynote characteristics that justify the strong interest for this annealing technique. In this work, we deal with the problem of the explosive crystallization that occurs in the presence of an amorphous layer, which is a very common condition in the usual doping process, and we apply an extended formulation of the phase field method in order to simulate the kinetic evolution during this phenomenon. We obtain a robust, reliable, and efficient method for the study of a three phase system with phase change, without introducing any additional ad-hoc variables. Our results have been compared with experiments in order to ensure an accurate calibration and verification of the numerical tool. This type of simulation is very interesting for the application in order to get complete information about the process which is difficult to achieve only with experimental characterization. Indeed, it is possible to predict if there is a melting or not, the melting depth and its duration, the temperature reached in the specimen as a function

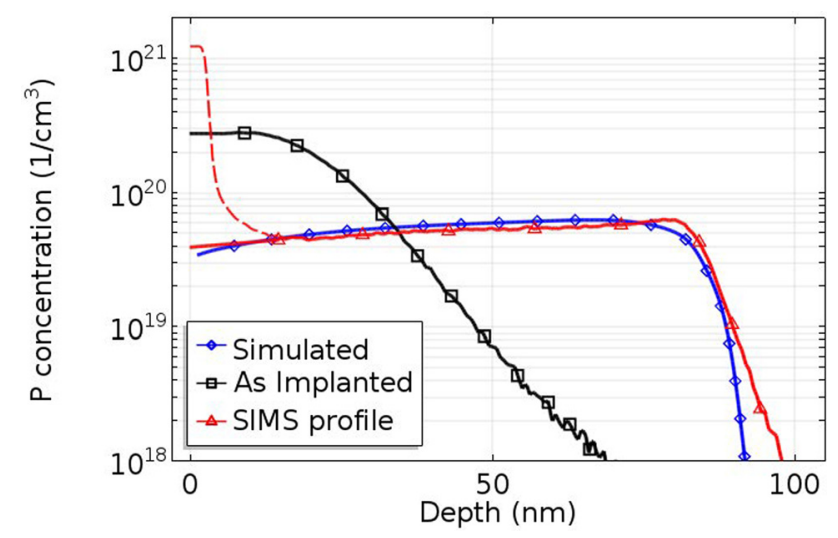

FIG. 10. Comparison between SIMS and simulation for the $1.2 \mathrm{~J} / \mathrm{cm}^{2}$ case. The black line with squares is the as implanted, red with triangles is the corresponding SIMS, and solid blue and rhombus is the simulated dopant curve. 
of the position, the evolution of the phases, and the dopant distribution. With this information, the miniaturization process could be more precise and specific.

Some topics need some refinements since the general application of the method, also in the case of device structure based on conventional materials like silicon or germanium, is not always straightforward and the far-from-equilibrium condition achieved by LA needs further study. In particular, a complete calibration of the optical parameters as a function of the laser frequency, temperature, and phases for any material used in nano-electronics is still missing. This is an issue for a self-consistent method due to the strong interaction and correlation between the laser heating and the structure local properties (geometry temperature, phase and dopant/alloy distribution). Moreover, the future implementation of the formalism in numerical tools able to simulate two and three dimensional geometries will give the possibility to simulate the lateral propagation of the EC phenomenon extending the potential prediction power of the method.

The authors acknowledge the cooperation project between CNR and CNRS "Understanding and Modeling of Excimer Laser Annealing” (UMEX).

${ }^{1}$ M. Tabbal, M. J. Aziz, C. Madi, S. Charnvanichborikarn, J. S. Williams, and T. C. Christidis, "Excimer laser processing of novel materials for optoelectronic and spintronic applications," Proc. SPIE 6458, 645803 (2007).

${ }^{2}$ V. A. Shklovski and V. M. Kuz'menko, Sov. Phys. Usp. 32, 163 (1989).

${ }^{3}$ V. P. Koverda, N. M. Boganov, and V. P. Skripov, "Explosive crystallization of amorphous substances with fixed crystallites," in Growth of Crystals, edited by E. I. Givargizov and S. A. Grinberg (Springer US, Boston, MA, 1991), pp. 69-81.

${ }^{4}$ G. Götz, Appl. Phys. A 40, 29 (1986).

${ }^{5}$ L. Nikolova, M. J. Stern, J. M. MacLeod, B. W. Reed, H. Ibrahim, G. H. Campbell, F. Rosei, T. LaGrange, and B. J. Siwick, J. Appl. Phys. 116, 093512 (2014).

${ }^{6}$ E. P. Donovan, F. Spaepen, D. Turnbull, J. M. Poate, and D. C. Jacobson, Appl. Phys. Lett. 42, 698 (1983).

${ }^{7}$ E. P. Donovan, F. Spaepen, J. M. Poate, and D. C. Jacobson, Appl. Phys. Lett. 55, 1516 (1989).

${ }^{8}$ M. Wautelet, Phys. Status Solidi B 159, K43 (1990).
${ }^{9}$ A. Filipponi and A. Di Cicco, Phys. Rev. B 51, 12322 (1995).

${ }^{10}$ P. Baeri, S. U. Campisano, M. G. Grimaldi, and E. Rimini, J. Appl. Phys. 53, 8730 (1982)

${ }^{11}$ M. O. Thompson, G. J. Galvin, J. W. Mayer, P. S. Peercy, J. M. Poate, D. C. Jacobson, A. G. Cullis, and N. G. Chew, Phys. Rev. Lett. 52, 2360 (1984).

${ }^{12}$ A. La Magna, P. Alippi, V. Privitera, and G. Fortunato, Appl. Phys. Lett. 86, 161905 (2005).

${ }^{13}$ O. Penrose and P. C. Fife, Physica D 43, 44 (1990).

${ }^{14}$ S.-L. Wang, R. Sekerka, A. Wheeler, B. Murray, S. Coriell, R. Braun, and G. McFadden, Physica D 69, 189 (1993).

${ }^{15}$ H. Udaykumar, R. Mittal, and W. Shyy, J. Comput. Phys. 153, 535 (1999).

${ }^{16}$ A. Karma and W.-J. Rappel, Phys. Rev. E 57, 4323 (1998).

${ }^{17}$ I. Singer-Loginova and H. M. Singer, Rep. Prog. Phys. 71, 106501 (2008).

${ }^{18}$ S. Lombardo, S. Boninelli, F. Cristiano, G. Fisicaro, G. Fortunato, M. Grimaldi, G. Impellizzeri, M. Italia, A. Marino, R. Milazzo, E. Napolitani, V. Privitera, and A. L. Magna, Mater. Sci. Semicond. Process. 62, 80 (2017).

${ }^{19}$ G. Fisicaro, L. Pelaz, M. Aboy, P. Lopez, M. Italia, K. Huet, F. Cristiano, Z. Essa, Q. Yang, E. Bedel-Pereira, M. Quillec, and A. L. Magna, Appl. Phys. Express 7, 021301 (2014).

${ }^{20}$ J. Bragard, A. Karma, Y. H. Lee, and M. Plapp, Interface Sci. 10, 121 (2002).

${ }^{21}$ H. Vogel, Phys. Z. 22, 645-646 (1921).

${ }^{22}$ G. S. Fulcher, J. Am. Ceram. Soc. 8, 339 (1925)

${ }^{23}$ S. Stiffler, P. Evans, and A. Greer, Acta Metall. Mater. 40, 1617 (1992).

${ }^{24}$ A. A. Wheeler, W. J. Boettinger, and G. B. McFadden, Phys. Rev. A 45, 7424 (1992).

${ }^{25}$ N. A. Ahmad, A. A. Wheeler, W. J. Boettinger, and G. B. McFadden, Phys. Rev. E 58, 3436 (1998).

${ }^{26}$ I. Trevi, J. M. Poate, G. Foti, and D. Jacobson, in Surface Modification and Alloying by Laser, Ion, and Electron Beams, edited by S. S. B. Media (Springer, Boston, MA, 2013), p. 414.

${ }^{27}$ P. Galenko, E. Abramova, D. Jou, D. Danilov, V. Lebedev, and D. Herlach, Phys. Rev. E 84, 041143 (2011).

${ }^{28}$ G. Fisicaro, K. Huet, R. Negru, M. Hackenberg, P. Pichler, N. Taleb, and A. La Magna, Phys. Rev. Lett. 110, 117801 (2013).

${ }^{29}$ I. Štich, R. Car, and M. Parrinello, Phys. Rev. Lett. 63, 2240 (1989).

${ }^{30}$ J. T. Okada, P. H.-L. Sit, Y. Watanabe, Y. J. Wang, B. Barbiellini, T. Ishikawa, M. Itou, Y. Sakurai, A. Bansil, R. Ishikawa, M. Hamaishi, T. Masaki, P.-F. Paradis, K. Kimura, T. Ishikawa, and S. Nanao, Phys. Rev. Lett. 108, 067402 (2012).

${ }^{31}$ N. Jakse and A. Pasturel, Phys. Rev. B 79, 144206 (2009).

${ }^{32}$ P. M. Fahey, P. B. Griffin, and J. D. Plummer, Rev. Mod. Phys. 61, 289 (1989).

${ }^{33}$ G. Fisicaro and A. La Magna, J. Comput. Electron. 13, 70 (2014). 